\title{
SAFETY DEVICE AND VEHICULAR AUTOMATION PROCESS BASED ON SPEED VARIATION
}

\author{
Authors: \\ Fausto Ferrer Fróes ${ }^{1}$ \\ Marcelo Soares de Carvalho ${ }^{2}$ \\ ${ }^{1}$ Avante Engenharia e Consultoria Ltda \\ ${ }^{2}$ Avante Engenharia e Consultoria Ltda \\ 1faustoffroes@yahoo.com.br \\ 2marcelocarvalhodesign@gmail.com
}

\begin{abstract}
This invention, for use in automobiles of all sizes, refers to an electronic device which automates the up/down feature of vehicles power-window systems, and simultaneously controls the air-conditioning, and/or any other electronic/electromechanical device connected to it as the vehicle's speed varies. This invention is meant to raise and lower windows as the vehicle speeds down and up to a preset speed, and simultaneously triggers other electronic devices connected to it, for example when the vehicle slows down to speeds below 20 $\mathrm{km} / \mathrm{hr}$. It has been designed to allow only the driver's window to lower as vehicles often circulate with no passengers. It may also switch off other electronic devices connected to it when the vehicle reaches speeds above $60 \mathrm{~km} / \mathrm{hr}$ (adjustable preset speed). Additionally, the invention has been designed to exchange intra and extra vehicle information solutions by means of a wireless network and GSM/GPRS. Such feature has not yet been developed by any prior art as verified by searches carried out in the patent database, which showed that no patent has brought forth a technological solution featuring identical functions and purposes of this invention. Once enabled, the device will be activated in a variety of situations, such as, for example, when approaching traffic red lights, in slow traffic, at night, or when approaching speed bumps, tollbooths, crossing and turning point, driving on rough roads, entering drive-throughs, always ensuring safety to vehicle occupants.
\end{abstract}

\section{INTRODUCTION}

This Safety Device and Vehicular Automation Mechanism has been designed to be automatically switch on/off as the vehicle speed changes, and it's meant to be used by the automobile manufactures in vehicles of any size. It's an electronic safety device that controls the power-window system to raise and lower the windows, and simultaneously controls the air-conditioning, or any other electronic device connected to it depending upon the vehicle speed variation.

This invention introduces an electronic safety solution in both situations, standstill and motion, ensuring comfort to the drivers and riders at a low cost since the described device makes use of built-in standard vehicle hardware, network and the power-window system. It's a cutting-edge technologic proposal based on Artificial Intelligence comprised by 
microcontrollers, DSPs or ASICs that allows the user to set it up by entering the User Menu, which makes it more versatile. Optionally, the device may be integrated to a human-machine interface system such as natural language user interfaces (LUI or NLUI), a facial recognition system, an eye tracking interface, and autostereoscopic interface aimed to improve vehicle's data safety and CAN (Controller Area Network). This article is structurally based on the Letters Patent Nr. PI 1100518-1 issued on Apriel 4th, 2017.

\section{GENERAL INFORMATION}

This device has been designed to allow users to raise and lower vehicle's power-windows, and simultaneously handle other electronic and electromechanical devices and components connected to it when the vehicle will reach speeds below $20 \mathrm{~km} / \mathrm{h}$ (suggest preset speed). It also allows to lower on the driver's window as most of the times vehicles circulate with no riders, and the reconfiguration and/or deactivation of electronic devices and components connected to it, when the vehicle reaches speeds above $60 \mathrm{~km} / \mathrm{h}$ (suggested preset speed).

Currently, automobiles are equipped with many controllers called ECU (Electronic Control Unit) that perform the electronic management of many vehicle systems such as, for example, EFI (Electronic Fuel Injection), air bag, air-conditioning, ABS break systems, alarms, windows, power door locks, radios, auto designed to provide comfort. ECUs may work independently without communication between them, or may communicate with many ECU set by specific networks designed by the automobile industry such as, for example, Controller Área Network ( CAN bus) connected by two wires that interconnect all Automotive Network Architecture for ECUs Communications and Subnetworks such as LIN (Local Interconnect Network), MOST network (Media Oriented Systems Transport), among others. Every type of network works based on specific protocol standards, types and data transmission speed, logic level handling, and others.

Such networks allow, for example, information exchange and share of signals and sensor codes. Each ECU work similarly, i.e., they receive data from sensors, interpret and send signals to actuators that perform many tasks such as, for example, triggering the air bag during a collision or impact, or set up the AIS Automatic Idle Speed by receiving RPM sensor signals. Regarding power-window systems, newer vehicles feature electromechanical systems comprised by electrical motors and some levers and/or steel cables installed inside vehicle doors to raise and lower power windows. The described invention is represented in a diagram by system blocks as shown in Figure 1. 


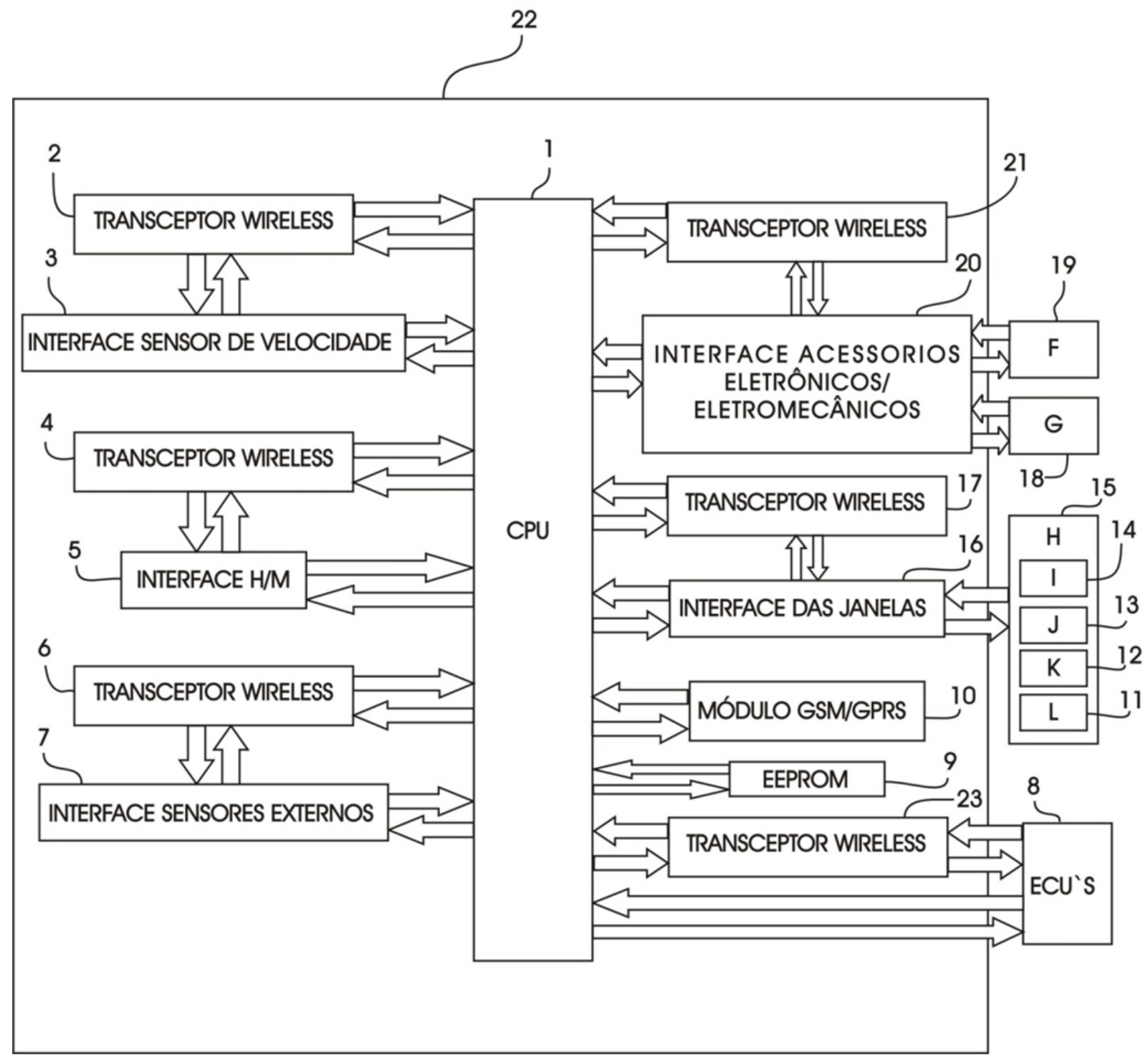

Figure 1 - Diagram of system blocks.

Figure 1 shows the diagram of system blocks of this invention usually indicated by the reference number 22. The encoder pulses are detected by a pulse detector circuit of the speed sensor interface (3) that generates representative normalized voltage signal pulses (Vc). These pulses are captured and counted by the microprocessor of the processing unit (1), and based on such counting, it calculates the vehicle speed based on its instant speed. The microprocessor receives the digital code corresponding to the input programming variables by the human-machine interface (5) that's set by the operator. The Input programming variables indicate highest and lower speed, upper and lower set points that determine the raising and lowering the power windows by the windows interface (16), and select possible programming for turning on and off the devices F and G (18) and (19) connected to the system. The microprocessor (1) sends control signals that change the logical state of its plugs connected to the inputs of the circuits of the windows interface (16) and other devices (20) when the speed signals are received by the microprocessor (1) reaches some of the values programmed in the human-machine interface (5) that may features keyboards, LCD screens, organic lightemitting diode (OLED) screen, touch screen, Voice Activated Switch, biometric authentication for access control of different types, i.e., biometric fingerprint, iris and face recognition, with no restriction. GSM/GPRS module (10) and Wireless (2), (4), (6), (17), (21) and (23) wirelessly transmit and receive all data managed by the microprocessor (1). The external sensor interface (7) receive surroundings data from smart (or not) sensors for 
dynamic construction of computing models to control, set up and adjust the current application, and send them to the microprocessor (1).

This device is divided int 9 basic sections, i.e., human-machine interface, sensor signal input interface or pulse detector (3), input interface to connect external sensors (7), wireless transceptor (2, 4, 6, 17, 21 and 23), GSM/GPRS module (10), processing unit (1), powerwindow circuit interface (16), power drives of auxiliary devices or power-window circuit interface bus (20), and EEPROM Memory (Electrically-Erasable Programmable Read-Only Memory (9).

The device interacts and exchange information with the ECU H (15) that control the windows and other ECUs (8) of the vehicle by wired or wireless connection to the signal inputs of the interface bus (3), and it's connected to the data bus that conveys information from the speed sensor to the ECU, gateway (8). Information external sensors are connected to the external input interface bus (7). The circuit bus of the power-window interface (16) is connected to input bus or directly to cable bunches that convey information to each switch to raise powerwindows I, J, K, L (11, 12, 13 and 14 to ECU H (15), which controls power-windows. Inputs/Outputs of the electro/electronic and electromechanical devices (20), the channels for setting up parameters for electro/electronic devices to be controlled, and also the electrical grid to connect other possible electronic and electromechanical devices with on/off switch of the vehicle. The human-machine interface or user's interface (5) is a set of features by which the user interact with machines, devices, computing software or any othe complex tool. It provides the means by which the users to handle the technology offered by means of an input that allows the user to operate the system by means of an output that command the system to perform an action requested by the user. It's possible to use many user's interface, with no restriction.

With the increase of data transmission capacity, the communication and interaction between humans and machines through electronic interfaces (5) have become more and more powerful, and have provided interfaces that explore more deeply the user's sensory and motor organs, and making system language more friendly and intuitive to its users, and making human-machine interaciton and human usability easier, quicker, more ergonomic. All that, take into account the physical and mental components of human activities, and as well its cognitive structures related to reality perception that, by sensory information, detects, identifies and interprets signals.

This invention presents as a suggestion, the use of HMIs (5) such as voice recognition and command, face detection, iris tracking interface and autostereoscopic interface. Vokce recognition and command interface used artificial intelligence, also called machine learning to recognize the natural language, based on computing models and specific algorithms. Such recognition is done through a microphone connected to the interface bus input, which converts voice analog signals into digital signals by using an analog-to-digital converter. These files are converted into text files or commands. By using the Fourier series and/or many mathematical techniques, a recorded voice tone is compared to the live voice of the person being identified. Such recognition will be used to enable, or not, the system. The data collected by the voice recognition interface are encoded and sent to the device microprocessor in form of digital data and commands.

Face detection and recognition module use a machine vision system based on artificial intelligence. Machine vision system comprises hardware and software that allow for capture, save and edit images, and can also recognize, identify or verify a person from a digital image or a video frame from a video source by using infrared rays to capture 3D images of objets. 
The rays are directed to the face of the person and then reflected backwards. The time difference tha light takes to return provides the system with a 3D object image, and that's the way the device receives and recognizes the image, which eases the decision making process, i.e., based on the visual authentication. The same system may be used for gesture recognition. The data collected by the face recognition module are encoded and sent to the microcontroller of the device in form of digital data and commands.

The iris tracking interface uses a biometric recognition system that emits a light that may be infrared, to enlarge the pupil. By making a vertical motion over the eyes, the system identifies dots and rings in the stained strip of the occular globe. The captured image is converted into mathematic representation, decoded by an algorithm to allow the device to compare it to other images previously stored. The system cross-check the mathematical result with other data to identify the individual among the other ones recorded in the database. Such data related to eye tracking are encoded and sent to the microcontroller of the device in form of digital data and commands.

The autostereoscopic interface are glass-free 3D screens that display 3D images. The technology used in this kind of display comprised an LCD screen or OLED (Organic LightEmitting Diode) display, a high speed screen and a convex lens and a series of confined space lighting in form of bars. The bars are lightened one at a time so that it's synchronized with the screen that displays the scene vision, laterally adjacent to each other, one at a time. Thus, the convex lens placed in front of this system behaves as a direction modulation so allowing the image vision to be only visible from only one direction depending on the viewer position. The screen must feature a high-frequency image update capability to provide for a better viewing results.

WSN sensors are smart wireless sensor networks that basically consist of a transceptor, a memory unit, pa rocessor, a sensor and a battery. These sensors are autonomous devices that feature sensory, processing and communication capabilities. When theses sensors (nodes) are networked in ad hoc mode, they form sensor networks. The nodes collect data, process it locally or coordinately among the neighbors, and may send information to the user or, in general, to a data sink. When many sensors are used, it's called WSN (Wireless sensor network). The main components of wireless sensor networks are the sensor nodes, communication wireless interfaces and nodes for communitation with other entities (gateway nodes). The WSN sensors used in this invention may behave as sensors and actuators, and are used as a resource to replace a great amount of interconnecting cabos in the internal structure of the vehicles. The use of this technology also allows these sensors to be shared to perform different functions in the vehicle, be connected by a satellite, and exchange data to provide control, safety adn monitoring of roads, and urban road networks.

This invention features an algorithm recorded by a signal processing unit comprised by a microcontroller/programmable processor (1) that receives from the vehicle speed sensor the pulses that determine the RPM numbers generated by the encoder of the vehicle. The microcontroller (1) count such pulses and, by an algorithm specially designed for this invention, record it in the microcontroller memory (1) to perform all functions provided by the device. The algorithm records the frequency of the pulses received and calculates the instant speed.

After processing the pulses received, the information from the sensors (7) and from the Human-Machine interface (5), from the Windows (16) and from the key of the windows ((11, $12,13,14)$, the system makes the decision to raise or lower the vehicle's power windowns and /or set up, switch on or off the electro-electronic and/or the electromechanical devices (18) and (19). To do so, the microcontroller (1) sends electrical signals tothe circuits of the interface of the windows (16) and/or to the interface of the electro-electronic and/or 
electromechanical devices (20) to perform data exchange and logic switching envolved in the process.

The functioning mechanism of this invention start from the time at which the device is switched on and the system scans its inputs and outputs to check whether there are pulses being sent from the speed sensor to the microcontroller (1).

The system calculates how many pulses arrive within a time interval, and convert them into instant speed. If the speed is lower than the upper set point, for example $60 \mathrm{~km} / \mathrm{h}$, the system remains in stand by mode, and if it's above $60 \mathrm{~km} / \mathrm{h}$ for more than 10 seconds, as reccomended, it enters the stage of power-window tracking, lowers the driver's window, and send a message to the HM interface (5) asking for authorizatin to switch off the electroelectronic devices. If the driver presses the corresponding button authorizing it, the system will optionally switch off the devices connected to it.

If the window up/down switch has been pressed by the driver, the system will remain in standby mode, and if the driver has not pressed the up/down switch, the microcontroller (1) will send electrical signal to the circuit of the interface of the windows (16) and/or to the electro-electronic interface (20), which will effect the automatic switching, raise the driver's windows, and switch off the air-conditioning, and/or optionally the devices connected to it.

After tracking the power windows, the system will remain in standby mode while the vehicle speed is above the lower set point, for example, $20 \mathrm{~km} / \mathrm{h}$. If the vehicle speed remains below $20 \mathrm{~km} / \mathrm{h}$ for more than 3 seconds, as a suggestion, the microcontroller (1) will send electrical signals to all switching circuits that account for lowering the all power windows. If any window is intercepted when it's been raised, the system will send a message to the HumanMachine interface (5) requesting it to stop raising it. Consecutively, the system will send a message to the HM interface requesting authorization to switch on the electro-electronic devices and, if authorized by the respective electro-electronic device of the HM inteface, it will switch on all devices connected to it, and will rescan the system from the beginning, and so it will complete the whole algorithm cycle.

In slow traffic condition, it's usual the vehicle to move at speeds below $60 \mathrm{~km} / \mathrm{h}(20<$ and $<60$ ), and in such a situation, it may reach speeds above $20 \mathrm{~km} / \mathrm{h}$ (as a suggestion), however without reaching the speef of $60 \mathrm{~km} / \mathrm{h}$ (as a suggestion), and once more, speed down to speeds below $20 \mathrm{~km} / \mathrm{h}$. In such a situation, to prevent this invented device from triggering the powerwindow system to raise the windows, and lower the driver's windows, and as well switch on or off the electro-electronic devices connected to it repeatedly at short intevals, it's a must to observe the following procedures:

A) If the vehicle has speed down to a speed below $20 \mathrm{~km} / \mathrm{h}$ (as a suggestion), and the device has been switched on and them back to the standby mode, it will only be switched on again, when the vehicle has reached a speed above $60 \mathrm{~km} / \mathrm{h}$ for more than 10 seconds (as a suggestion). Only after that, it will be able to be switched on when the vehicle will speed down to a speed below $20 \mathrm{~km} / \mathrm{h}$ for 3 more seconds (as a suggestion). Then, the device will remain idle in standby mode, and the the power-window system could be manually switched on by the manual switches, and as well the electro-electronic devices by their respective manual switches.

B) It will also happen, if when the vehicle reaches speeds above $60 \mathrm{~km} / \mathrm{h}$ for more than 10 seconds (as a suggestion), i.e., the device will be switched on, perform its operation and return to standby mode. The vehicle must speed down to a speed below $20 \mathrm{~km} / \mathrm{h}$ for 3 seconds (as a suggestion) to be ready to be switched on again, and once more able to operate when the speed reaches more than $60 \mathrm{~km} / \mathrm{h}$ (as a suggestion). If it won't happen, i.e., if the speed will not 
reach below 20km/h (as a suggestion), the device will remain idle in standby mode, and the windows will be only raised and lowered by pressing the manual switches, while the electroelectronic devices by their own manual switches. Therefore, to predetermine a speed below $20 \mathrm{~km} / \mathrm{h}$ (as a suggestion), as soon as it's reached, the device, by means of its electronic switching circuit will raise all power windowns, and will switch on the electro-electronic devices connected to it such as the air-conditioning, and the driver's window will be lowered, while other electro-electronic devices will be switched off, if there's any. When the vehicle speeds up and reach a preset speed above $60 \mathrm{~km} / \mathrm{h}$ (as a suggestion).

It's a safety device, and it's respective mechanism designed to automate the power-windows system based on the vehicle speed in which a pulse detector circuit detects the inputs released by a vehicular encoder, and send such pulses to one of the inputs of a microcontroller ou microprocessor. (1) An HM interface (5) communicates with the microcontroller to select the input programming. An interface circuit communicates with the microcontroller (1), receives controlling signals from the microcontroller (1) and send such signals to the ECU (5) that control the vehicle's power-windows system and/or other ECUs (8). Another interface circuit (20) also communicates with the microcontroller (1), receives controlling signals from the microcontroller (1), and switches on other electro-electronic devices and/or electromechanical devices connected to such an interface circuit (18 and 19).

Nevertheless,the microcontroller (1), by a software installed in its internal memory and/or EEPROM (9), interprets teh number of pulsed received, converts them into instant speed, and determine the controlling signal transmission to the two interface circuits, which controls the power window to be raised and lowered and/or switches on and off other devices connected to it. Every data exchange between several stages of teh device may be done by physical means, by wired and wireless communication, which potentializes the protocols and technology applications in telematics, X-by-wire, processing smart sensors, pervasive computing (Ubiquitous Computing), and intravehicular communication.

Wireless transceptors modules (2), (4), (6), (1), (21), and (23) are present at all stages. They may use many available wireless transceiver tecnonologies such as Bluetooth (IEEE 802.15.1), Bluetooth (IEEE 802.15.1), UWB - Ultra Wide Band (IEEE 802.15.3a), Wi-Fi (IEEE $802.11 \mathrm{a} / \mathrm{b} / \mathrm{g}$ ) and others, depending on the sensory application type. The system features an interface (7) for many types of sensors such as for sensing temperature, humidity, luminosity, wireless WSN, with no restrictions. The system also features a GSM/DPRS to access TCP/IP, mobile and telematic telephony.

By using (5) Human-Machine interface (5), a touch screen, for example, the driver will have mobility to switch it on and off, and quickly and effectively change previous system settings. Therefore, this device features a diversified application field by the automobile industry as it may be installed in any automotive vehicle equipped with a power-window system.

Traffic statistics in Brazil and abroad shows a high rate of crimes committed by the time a vehicle is getting close to red traffic lights, slow traffic situations such as traffic jams, night driving situations, and/or by approaching road speed bumps, turnpikes, crossings, rough roads, turn points, entering drive-throughs and other similar situations.A simple preventive procedure may be taken whenever the vehicle is about to speed down, or has come to a complete standstill. Today, such procedure is done manually by pressing power-window system switches, usually located on the door panels, consoles, and more recently, on the steering wheels.

This manual system often favors theft of vehicle and its occupants as it takes time to switch on all up/down manual switches, usually under stress, and at some risk as the hands will be busy with other vehicle operations. This invent is meant to prevent such situation from happening. 
Besisdes providing safety to the vehicle occupants, this invent also provides comfort to the users as it provides information on the surroundings, and help build computational models to control, configure and adjust the application, and allow for better mobility by means of mobile computing, interconnection of portable devices such as smartphones, laptops, MP3 players, and more, so that it will create a vehicular environ to provide more comfort, safety and relaxation to its occupants in a integrated and smart way. It also provides automatic airconditioning switch on/off, and fuel economy by saving engine power as the air-conditioning will be automatically switched off whenever the driver's window is lowered.

Besides the advantages already mentioned, this invent also provides easy different triggering ways by a single command that may be tactile such as touch screen, mechanical command, sonorous signal, and more; it may be installled in optional places in the vehicle; it will also prevent the user from pressing a number of existing manual switches to raise and lower the power windows; it will not make any change in the other components of the vehicle; it's complementary and compatible with the existing manual up/down switches of conventional power-window systems; it won't overburden the preinstalled power-window system; combined with automotive window film, it will make it even more efficient; it will shorten the time for raising and lowering power windows; it may be enabled and disable at anytime by raising power windows, and turning on the air-conditioning and/or the other devices before the vehicle reaches a speed down to a speed below $20 \mathrm{~km} / \mathrm{h}$ (as a suggestion); or lower the driver's window, turn off the air-conditioning and/or other devices before the vehicle reaches a speed above $60 \mathrm{~km} / \mathrm{h}$ (as a suggestion); it prevents possible heating of manual switches; it may be programmed to operate in predetermined situation; it may help natural ventilation inside the vehicle by lowering the driver'a window when the vehicle reaches a preset speed; it creates micro-ambiences inside the vehicles, and provide thermal comfort, or otherwise may provide other functions such as opening/closing the sunroof, convertible roof, massaging seats, and more; it may contribute to fuel and battery economy by switching off the airconditioning and other electro-electronic devices connected to it, when the vehicle reaches a preset speed; it prevents heating, if the air-conditioning is turned off, if it's the case, with the driver's window lowered; it allows improves engine power by turning off the airconditioning and other devices connected to it; it optionally allows for connecting to it the air-conditioning, fan, radio, CD/DVD/MPs players, convertible roof, sunroof, and other electro-electronic devices to improve comfort, entertaining devices, automobile workstation for vehicle occupants; it improves safety to the vehicle users by automatically raising all power windows at the same time triggered by just one command.

Today, the manual switching of power-window systems requires the user to press a number of single switches to fully raise and lower all power windows. Currently, raising and lowering the power windows require the users to press and hold single switches for certain time, otherwise the windows may be raised only partially, which's somewhat risky in situation such as mentioned above. It also reduces significantly the job of handling many single switches to make power windons go up and down, and trigger other electro-electronic devices currently available in the vehicles by automating power-window systems and other devices that may be connected to this invention based on the speed variation of the vehicles. 
The operation and process presented by this invention are itemized below and shown in the flowchart in the Figure2, where:

Start - Refers to the begining of the process;

S1 - Reads HMI - Human-Machine Interface;

S2 - Reads sensor signals;

S3 - Enable and configure windows and devices;

S4 - Standy by, countdown timer;

S5 - Speed above the upper set point?

S6 - Has the driver's switch been manually pressed?

S7 - Speed below the lower set point for more than 3 seconds?

S8 - Has the driver's switch been manually pressed by the driver?

S9 - Raise previously programmed power windows;

S10 - Reads sensor signal from the windows;

S11 - There was any interception of any window?

S12 - Sends message to HMI that determine which window has been intercepted;

S13 - The HMI requests authorization to switch on electro-electronic devices and/or previously programmed electromechanical devices;

S14 - Reads HMI;

S15 - Has the switch been pressed to authorize switching on the electric-electronic previously programmed devices (Yes/No)

S16 - Switches off the electro-electronic and/or electromechanical device(s)? ;

S17 Switches on electro-electronic and/or electromechanical previously programmed devices;

S18 - Speed above the upper set point for more than 10 seconds?

S19 - Driver window switch has been pressed?

S20 - Standy by, countdown timer;

S21 - Lowers previously programmed power windows;

S22 - HMI requests authorization to switch off electro=electronic and/or electromechanical previously programmed devices;

S23 - Reads HMI (Human-Machine Interface);

S24 - The switch has been pressed authorizing switching off electro-electronic and/or electromechanical previously programmed devices;

S25 - Keeps electro-electronic and/or electromechanical devices switched on;

S26 - Switches off electro-electronic and/or electromechanical previously programmed devices;

End - Resets the process to the starting point. 


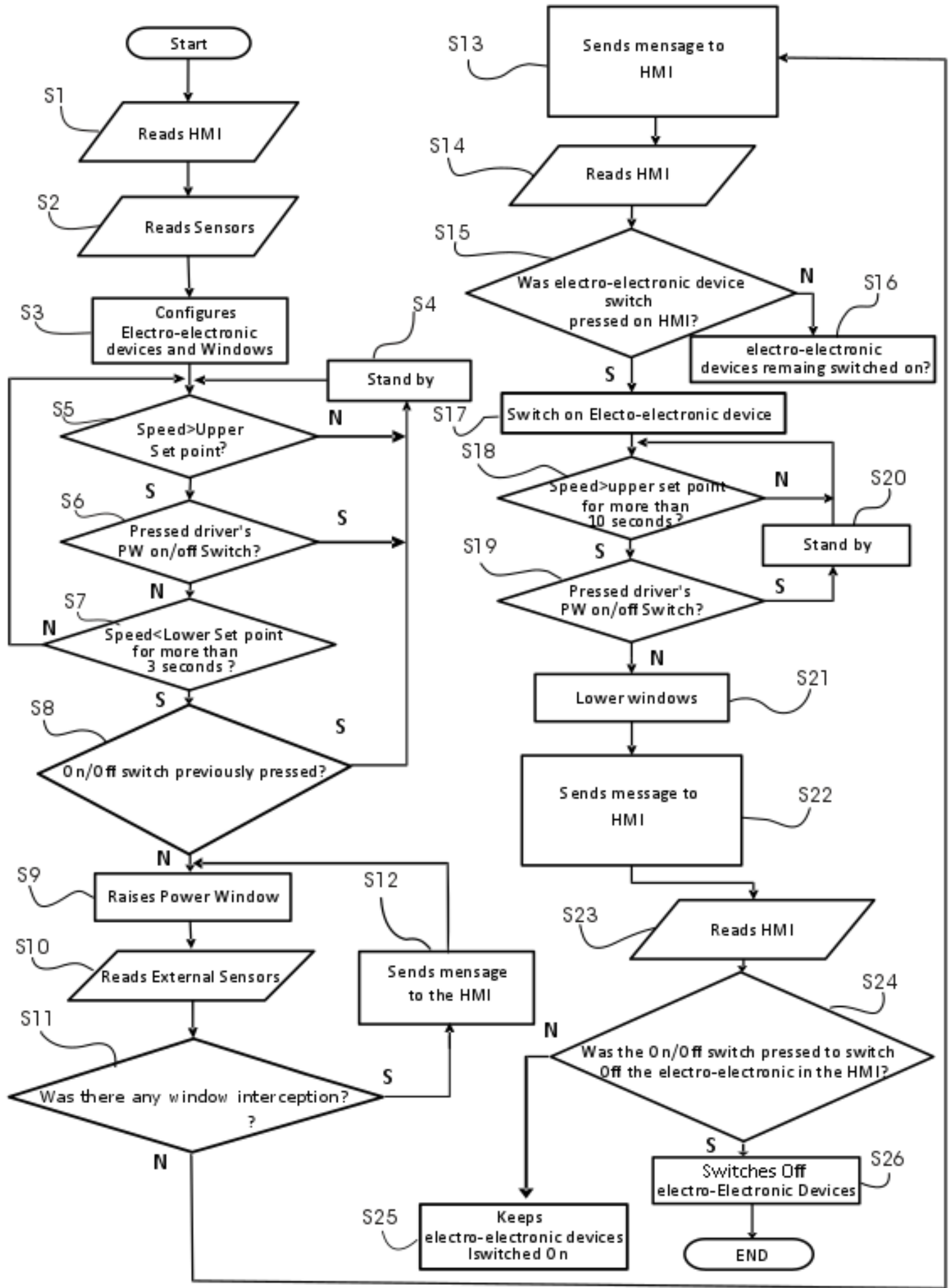

Figure 2 - Flowchart of general stages associated with the functioning process. 
The system features an algorithm specially designed for this invention, which is responsible for managing and running the process that's recorded in the processing unit (1) that has a programmable microcontroller/processor. The pulse detector (3) detects the signals from the speed sensor that determine RPMs generated by the vehicle encoder. The microcontroller (1) connnected to the pulse detector counts the pulses and calculates the instant speed by an algorithm exclusively designed for this invention, record it in the internal memory and/or EEPROM (9), and run all functions required by the device process as can be seen in the flowchart shown in Figure 2.

Block S1 reads and stores HMI configuration; Block S2 captures and store pulse signal data sent by the encoder and, concomitantly, calculates vehicle instant speed; Block S3 configure and/or enables windows and and devices programmed by the HMI (5); Block S4 keeps the system in Standby mode for a programmed period of time; Block 5 compares the instant speed value to the upper speed value (upper set point) previously programmed by the operator; Block S6 checks whether the on/off switch of the driver's power window has been switched on; Block S7 sends a controlling signal to the interface circuit of the power windows (16), if the recorded speed is below the lowest programmed speed for more than 3 seconds (as a suggestion) and the driver's window on/off switch has not been switched on by the Block S8, the microcontroller (1).

The power-window interface circuit (16) send a logic signal to the power-window ECU (15), which switches on the power-window system previously programmed in the HMI (5), and raise the previously programmed power windows according to the Block S9. In the Block S11 the system detects whether any of the power windows has been intercepted, and has been impeding it to be fully raised. In the Block S12, the system indicates to the driver by the HMI (5) which window has been blocked, and remains looping until the window is freed from any obstacle. In the Block S13, the system send a message by the HMI (5) requesting the authorization to switch on the previously programmed electro-electronic devices. In the Block S14, the HMI (5) is read in order to check whether the the switch corresponding to the electro-electronic devices envolved in the process has been switched on in teh HMI (5). In the Block 15, it's checked whether the switch (or switches) has been switched on or not and, if it's not been switched on, the electro-electronic devices will remain switched off according to the Block S16. In the Block 17, if the switch of the electro-electronic and/or electromechanical devices (20) have been switched on, they will be automatically switched on according to what's been previously programmed.

In the Block S18, if the speed recorded is above the upper speed (upper set point) previously programmed for more than 10 seconds, and the on/off switch has not been switched on, according to the Block S19, then the microcontroller (1) will send a controlling signal to the window interface circuit (16).

The window interface circuit (16) sends a logic signal to the power-window ECU (15) that switches the system on of the power window system previously programmed in tht HMI (5), and lower the power windows previously programmed according to the Block S21. Then, according to the Block S22, the system sends a message to the HMI (5) requesting autorization to switch off the previously programmed electro-electronic devices. The Block S23 reads the HMI (5) and the Block S24, if the it has not kept the electro-electronic devices switched on according to the Block 25. In the Block 26, if it has been switched on, the system will switch off the electro-electronic and/or electromechanical previously programmed (20), so ending the algorithm cycle, and returning to the beginning of the program. 


\section{CONCLUSION}

The device provides more protection and a feeling of safety to the users of vehicles equipped with power-window system by inhibiting criminal direct approaches so common nowadays, specially in the streets of large urban centers. Furthermore, it automates, rationalizes, optimizes and simplifies the use of existing comfort devices characterized by a variety of electro-electronic and electromechanical devices more and more provided by the automobile industry in the vehicles, however without compromising the user's and road traffic safety.

\section{REFERENCES:}

[1] BARONE, Dante. Sociedades Artificiais: A Nova Fronteira da Inteligência nas Máquinas. 1. ed. Porto Alegre: Bookman, 2003.

[2] CHARNIAK, Eugene; MCDERMOTT, Drew. A Bayesian Model of Plan Recognition. Massachusetts: Addison-Wesley, 1985.

[3] HAUGELAND, John. Artificial Intelligence: The Very Idea. Massachusetts: The MIT Press, 1985.

[4] LOUREIRO.Antonio, A.F; Redes de Sensores Sem Fio. Disponível em: www.dcc.ufmg.br/ loureiro/cm/docs/sbrc03.pdf.

LOPES, Silvana. Sistemas Especialistas na Educação. Ariquemes: Universidade Federal de Rondônia, 2008.

[5] MONARD, Maria Carolina; BARANAUKAS, José Augusto. Aplicações de Inteligência Artificial: Uma Visão Geral. São Carlos: Instituto de Ciências Matemáticas e de Computação de São Carlos, 2000.

[6] POOLE, D.; MACKWORTH, A. K.; GOEBEL, R. Computational Intelligence: A Logical Approach. Oxford: Oxford University, 1998.

[7] RUSSEL, Stuart; NORVIG, Peter. Inteligência Artificial. 2. Ed. Rio de Janeiro: Campos, 2004.

[8] STAIR, Ralph M.; REYNOLDS, George W. Princípios de Sistemas de Informação. São Paulo: Thomson, 2006

[9] FISCHLER, M.A; FIRSCHEIN, O. Intelligence: The Eye, the Brain, and the Computer. Addison-Wesley Professional, 1987.

[10] BARNLUND, D. C. A transactional model of communication. In: NORTENSEN, C. D. Communication theory. Brunswick, New Jersei: Transaction, 2008.

[11] TUFTE, E. Visual Design of the User Interface.Armonk, NY: IBM Corporation, 1989.

[12] CAPUANO, Francisco. G; IDOETA, Francisco Valeije. Elementos de Eletrônica Digital. São Paulo: Editora Érica.

[13] MICROCHIP. Automotive Recommended Product Selector Guide. Disponível em: http://ww1.microchip.com/downloads/en/devicedoc/00001320d.pdf . 